\title{
The Ultrasound of Perianal External Opening
}

\section{Youssef AT* \\ Department of Radiology, Faculty of Medicine, Fayoum University, Egypt}

*Corresponding author: Ashraf Talaat Youssef, Department of Radiology, Faculty of Medicine, Fayoum University, Egypt, Tel: 00201001849139; E-mail: ashraftalaat1@yahoo.com

\section{Research Article}

Volume 2 Issue 3

Received Date: October 03, 2017

Published Date: October 12, 2017

\section{Abstract}

Background: An abscess, fistula tract, fistula with abscess formation and sinus tracts can lead to perianal external opening. The commonest cause which comes to the mind of the clinicians when they observe perianal external opening(s) is the presence of perianal sepsis.

Methodology: 472 cases showing perianal external opening(s) were examined with transperineal, endorectal and if females with further transvaginal ultrasound.

Results: The incidence of anal abscess was 3.4\%, of perianal fistula was 93.4\%, pilonidal sinus tract extending to the perianal spaces was $1.5 \%$, the incidence of hiradenitis Suppurativa was $0.85 \%$, the incidence of infected epidermal inclusion cyst was $0.21 \%$ and Bartholin abscess was $0.64 \%$ among our study group.

Conclusion: Ultrasonography with its transperineal, endorectal and transvaginal approaches can be very helpful to the clinicians in the assessment of different etiologies that lead to the formation of perianal external opening to optimize the management.

Keywords: Ultrasonography; Perianal fistula; Hiradenitis Suppurativa

\section{Introduction}

An abscess, fistula tract, fistula with abscess formation and sinus tracts can lead to a perianal external opening (s). The commonest cause which comes to the mind of the clinicians when they observe perianal external opening (s) is the presence of perianal sepsis.

A perianal abscess can be perianal, submucosal, intersphincteric, ischiorectal or pelvirectal abscess [1]. Perianal fistula is a tract with lining granulation tissue showing an external opening and internal opening [2]. It can be classified according to the relationship between the fistula tracts and the anal canal sphincter muscles into, transsphincteric, intersphincteric, suprasphincteric and extrasphincteric fistula [3]. Perianal fistula can be calssified into 5 grades according to the severity and the way of management, where grade 1, 11 fistulas can be managed with fistulotomy, while grade $111-\mathrm{V}$ are complex fistulas that shouldn't be managed with fistulotomy [4].

Ultrasonography is a useful imaging diagnostic tool in evaluating and classifying the perianal fistula tract 


\section{Gastroenterology \& Hepatology International Journal}

according to the sphincteric course, extrasphincteric course, the site of internal opening, the varieties of the external opening, the etiology of fistula tract and the expected operative risks, through its transperineal, endorectal and transvaginal approaches in females [5]. Other uncommon etiologies reported to cause a perianal external opening rather than perianal fistula $[6,7]$.

\section{Aim of the work}

The current study aimed to assess the role of ultrasound assisted with three dimensional capabilities in evaluating, differentiating and estimating the incidence of different pathological processes that leads to the formation of the perianal external opening(s) among our study group.

\section{Methodology}

472 cases showing perianal external opening(s) were examined with transperineal, endorectal and if married females with further transvaginal ultrasound allover period of 8 years in the radiology department of my institute.

All patients with perianal sepsis showing no external opening were excluded from the study.

The age of patients included in the current study was ranged from 1 year to 70 years and the mean age was 31 years. They were 390 males and 82 females.

Their basal metabolic index (BMI) was ranging from 18 to 34 and the duration of symptoms ranged from 1 month to 50 months.

All were examined with Sonoace $\mathrm{x} 8$ ultrasound machine (Medison, Korea) and GE logiq 7 ultrasound machine (USA).

Among our study group, 6 pediatric patients were examined only with transperineal ultrasound using superficial linear probe and all other patients were examined with linear, endorectal and if married females endovaginal high frequency probes.

All patients were examined in left lateral decubitus positions with the knee and hip joints flexed.

We examined the site of the external opening (s) with transperineal ultrasound using the superficial linear probe for the presence of underlying abscess, sinus or fistula tracts.

The abscesses were evaluated for its size, extensions and relation to the anal sphincters and if any communicating sinus or fistula tracts.

The sinus tracts were evaluated for the site, length, width, extensions, side branching, the echo characteristics of its contents and the relation to the anal canal walls and anal sphincters.

The fistula tracts were evaluated for the site, length, side branches, the site of internal openings above, at or below the dentate line, and the relation of the fistula tract to the anal canal sphincters.

Further endorectal (and/or) endovaginal ultrasound was used to confirm the relationship between the fistula or the sinus tract and the anal canal sphincters.

The final diagnosis was reached based on the surgical results.

\section{Results}

Among 472 patients with perianal external opening (s), 16 cases detected with anal abscess (Figure 1), 441 patients with perianal fistula ( Figure 2a, Figure 2b \& Figure 2c), 7 patients with pilonidal sinus tract extending to the perianal spaces (Figure 3a \& Figure 3b), 4 cases with hiradenitis Suppurativa. (Figure 4a \& Figure 4b).1 case with infected epidermal inclusion cyst and 3 cases with Bartholin abscesses.

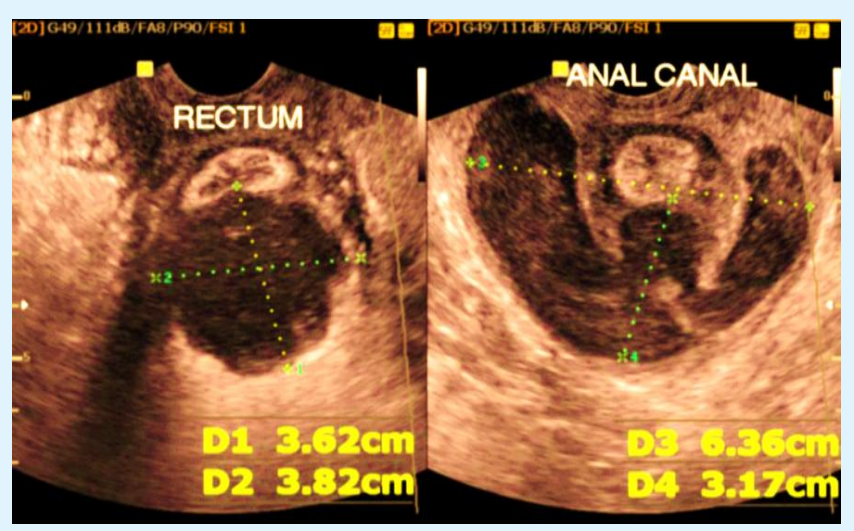

Figure 1: 2 dimmensional endovaginal ultrasound showing large posterior horse-shoe intersphincteric abscess seen extending from mid anal canal to the posterior rectal wall. 


\section{Gastroenterology \& Hepatology International Journal}

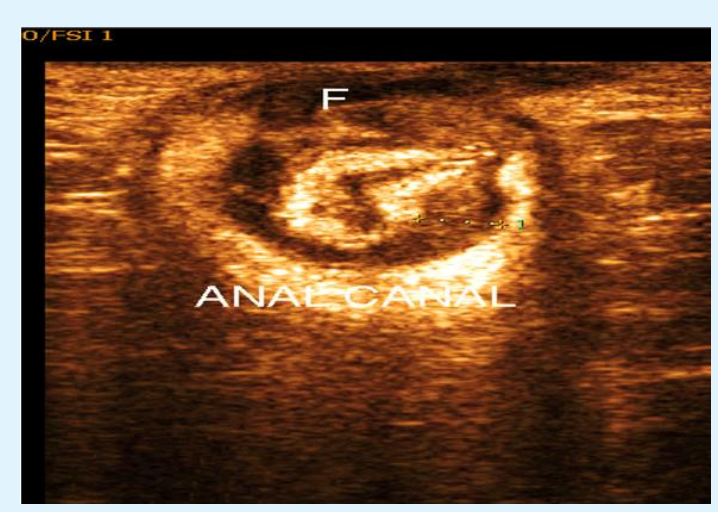

Figure 2a: Endovaginal ultrasound showing anterior transsphincteric perianal fistula (F).

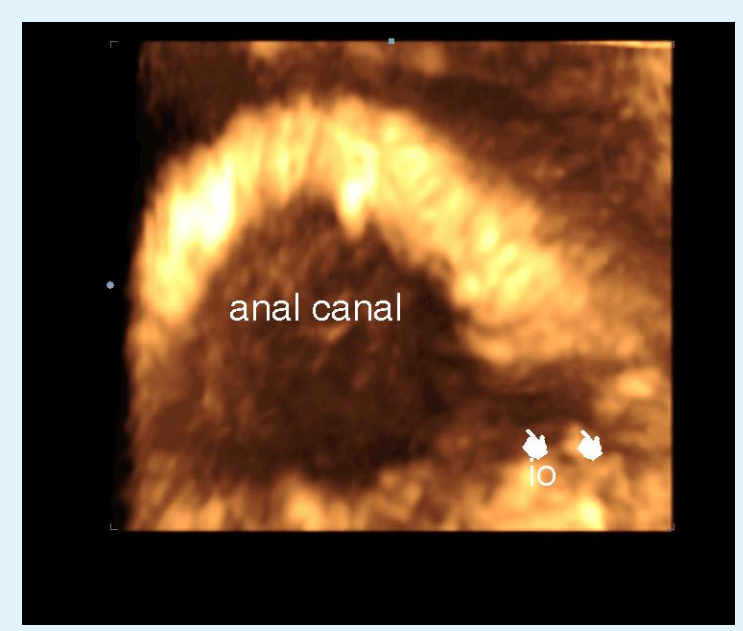

Figure 2b: 3 dimensional ultrasound showing the internal opening of perianal fistula.

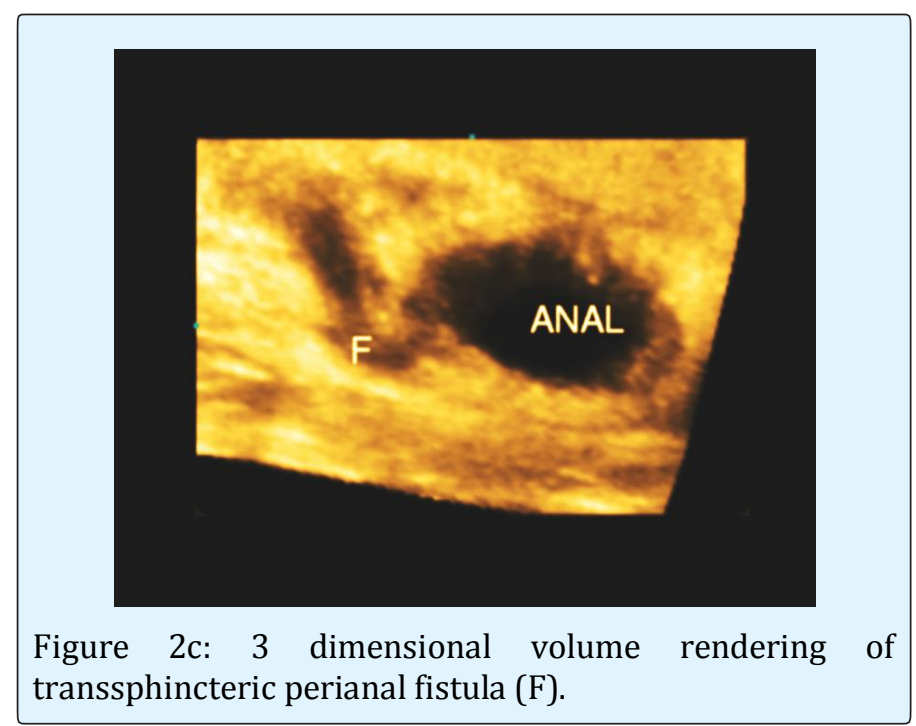

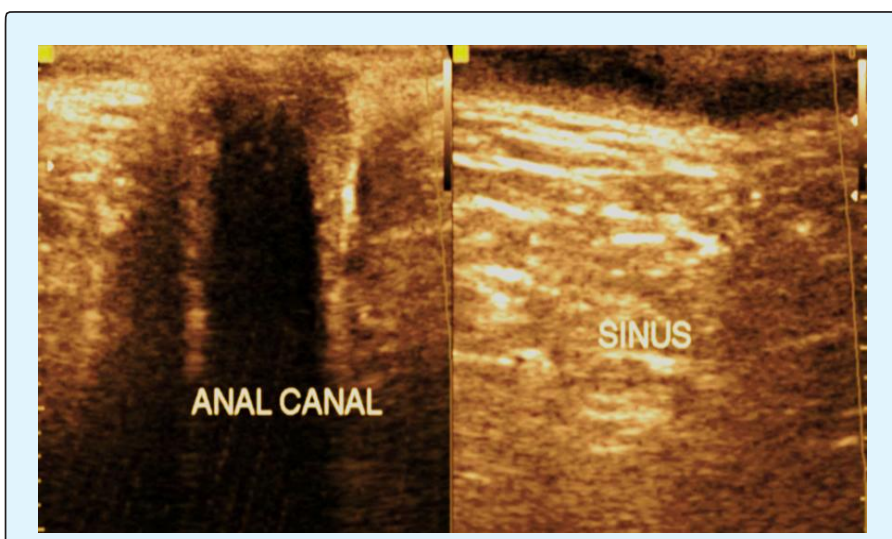

Figure 3a: Transperineal ultrasound showing pilonidal sinus tract extending to the perianal region

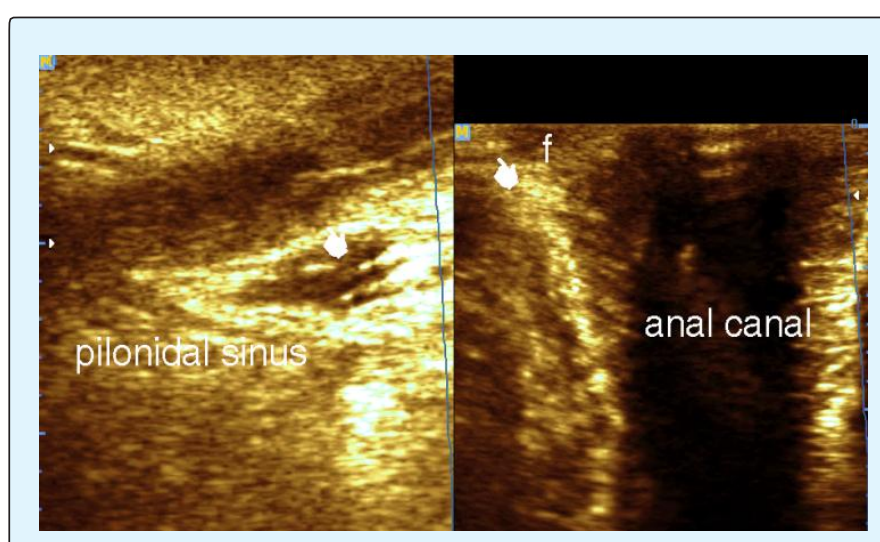

Figure 3b: Transperineal ultrasound showing pilonidal sinus extending deeply in the perianal region giving rise to low level perianal fistula

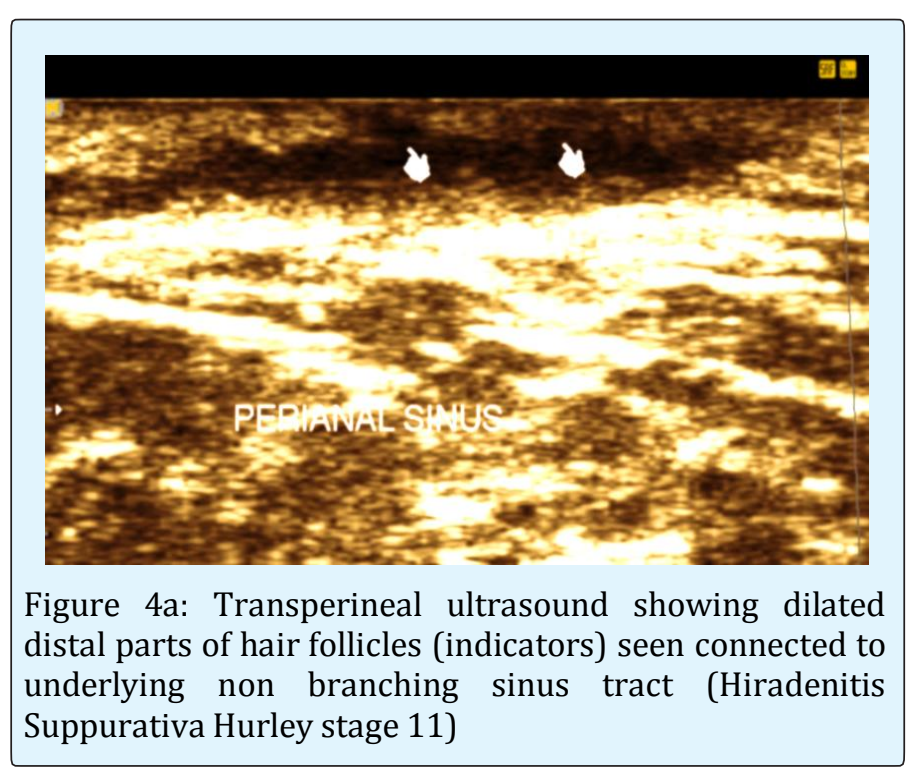




\section{Gastroenterology \& Hepatology International Journal}

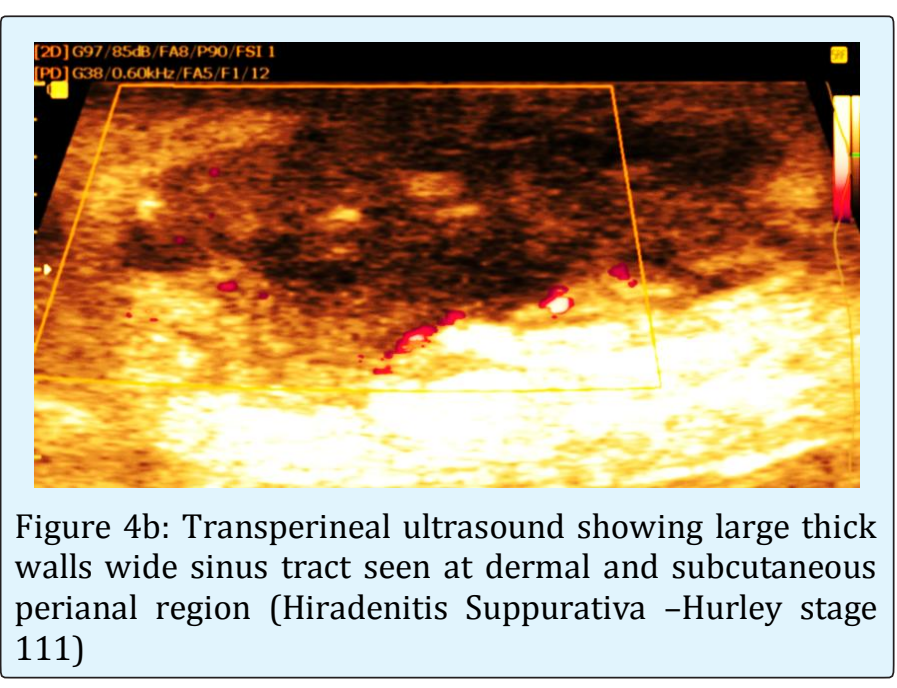

The incidence of anal abscess was 3.4\%, of perianal fistula was $93.4 \%$, pilonidal sinus tract extending to the perianal spaces was $1.5 \%$, the incidence of hiradenitis Suppurativa was $0.85 \%$, the incidence of infected epidermal inclusion cyst was $0.21 \%$ and Bartholin abscess was $0.64 \%$ among our study group.

Among patients diagnosed with anal abscesses, 5 patients showed a perianal abscess, 3 cases with interspincteric abscess, 6 patients showed ischiorectal fossa abscesses, 1 case showed horse-shoe abscess and 1 case with pelvirectal abscess.

Among patients with perianal fistulas, 333 cases detected with cryptoglandular perianal fistulas, 90 patients with recurrent cryptoglandular perianal fistulas and 18 patients with non cryptoglandular perianal fistula. Submucosal fistula was diagnosed in 7 patients, transphincteric fistula was diagnosed in 310 patients, intersphincteric fistula was diagnosed in 107 patients, extrasphincteric fistula in 9 patients and superficial fistula detected in 9 patients.

The sensitivity of ultrasound in diagnosing different types of fistula compared with the surgical results was $100 \%$ for superficial fistula, $87 \%$ for submucosl fistula, $92 \%$ for high transphinctericc fistula, $100 \%$ for low transphincteric fistula, 78\% for extrasphincteric fistula, and $96 \%$ for an intersphincteric fistula.

Among 4 patients with hiradenitis Suppurativa, 3 patients were classified as Hurley stage 11, and 1 patient as Hurley stage 111.
Among 7 patients with deeply extended pilonidal sinus tracts with an external opening in the perianal region, one external opening seen leading to superficial perianal fistula and one external opening seen leading to transsphicteric perianal fistula and both tracts were differentiated from the cryptoglandular perianal fistula by their extension to the natal cleft region and by the presence of echogenic lines inside suggesting of hair.

A case with infected epidermal inclusion cyst was seen among our patients showing subcutaneous cystic swelling with echogenic debris inside at the perianal region and was deeply extending to reach opposite the mid anal canal wall.

3 females were observed with irregular perianal subcutaneous thick walls cystic swellings showing turbid contents and marginal hyperemia with color Doppler study and were diagnosed as bartholin abscesses.

\section{Discussion}

Ultrasonography was a useful tool in distinguishing between the different etiologies of perianal external opening(s). Perianal fistula can be diagnosed by the presence of an external opening, an internal opening within the anal canal wall and a joining tract. The fistula tract can be none branching or branching, the site of internal opening can be detected with endoanal ultrasound whether at the dentate line or off the dentate line which helps to differentiate fistula of cryptoglandular origin from non cryptoglandular fistula. Recurring and non cryptoglandular perianal fistulas tend to be branching, high level and their internal openings situated off the dentate line and more liable for an abscess formation [8]. Anal abscesses are enclosed cavities of convex borders or concavo-convex borders with turbid echoes of pus debris inside showing marginal hyperemia and distal acoustic enhancement; it may show a communicating narrow tract with the internal opening [5] or communicating sinus or fistula tract with the external opening.

Pilonidal sinus showing deep extension into the perianal spaces usually doesn't communicate with the anal canal wall except in 2 cases of our 7 cases; it extends to the intergluteal region (natal cleft) and shows echogenic lines of hairs within.

Hiradenitis Suppurativa in the perianal region shows dilated distal parts of the hair follicles that communicate with underlying sinus tracts situated in the dermis and 


\section{Gastroenterology \& Hepatology International Journal}

subcutaneous region (Hurley stage 11) and in advanced stage the sinus tracts are more dilated, multiple and may show an abscess formation (Hurley stage 111) [7,9], the sinus tracts usually don't communicate with anal canal walls.

The current work emphasizes that not only the perianal sepsis can lead to perianal external opening in spite being the commonest. Other etiologies exist in spite uncommon; the clinician should consider all causes when he faces an external opening in the perianal region. Ultrasonography is a valuable investigating imaging modality that helps the clinician in reaching the etiology of perianal external opening and in the assessment of the disease extent.

\section{Conclusion}

Ultrasonography with its transperineal, endorectal and in female's transvaginal approaches can be very helpful imaging modality, in the assessment of different etiologies that lead to the formation of perianal external opening to optimize the management.

Conflict of Interest: The author declares that he has no conflict interest.

\section{Sources of Funding: not present}

\section{Informed Consent}

All procedures performed were in accordance with the ethical standards of the responsible committee on human experimentation (institutional and national) and with the Helsinki Declaration of 1975 (revised version of 2000) [5].

All patients provided written informed consent to the enrollment in the study and to the publication of information that could potentially lead to their identification.

\section{References}

1. Janicke DM, Pundt MR (1996) Anorectal disorders. Emerg Med Clin North Am 14(4): 757-880.

2. Abbas MA, Jackson CH, Haigh PI (2011) Predictors of Outcome for Anal Fistula Surgery. Arch Surg 146(9): 1011-1016.

3. Parks AG, Gordon PH, Hardcastle JD (1976) A classification of fistula-in-ano. Br J Surg 63(1): 1-12.

4. Garg P (2017) Comparing existing classifications of fistula-in-ano in 440 operated patients: Is it time for a new classification? A Retrospective Cohort Study. Int J Surg 42: 34-40.

5. Youssef AT (2015) Imaging Classification of Perianal Fistula Using the Ultrasound. Journal of Gastroenterology and Hepatology Research 4(6): 1653-1659.

6. Iqbal CW, Gasior AC, Snyder CL (2012) Pilonidal Disease Mimicking Fistula-in-Ano in a 15-Year-Old Female. Case Rep Surg 2012: 310187.

7. Wortsman X, Jemec G (2013) A 3D ultrasound study of sinus tract formation in hidradenitis suppurativa. Dermatol Online 19(6): 18564.

8. Loungnarath R, Dietz DW, Mutch MG, Birnbaum EH, Kodner IJ, et al. (2004) Fibrin glue treatment of complex anal fistulas has low success rate. Dis Colon Rectum 47: 432-436.

9. Hurley HJ (1989) Axillary hyperhidrosis, apocrine bromhidrosis, hidradenitis suppurativa, and familial benign pemphigus: surgical approach. In: Roenigk RK, et al. (Eds.), Dermatologic surgery. Marcel Dekker, New York, pp: 729-739. 